\title{
Executive working memory load does not compromise perceptual processing during visual search: Evidence from additive factors analysis
}

\author{
Jibo He And Jason S. McCarley \\ University of Illinois at Urbana-Champaign, Urbana, Illinois
}

\begin{abstract}
Executive working memory (WM) load reduces the efficiency of visual search, but the mechanisms by which this occurs are not fully known. In the present study, we assessed the effect of executive load on perceptual processing during search. Participants performed a serial oculomotor search task, looking for a circle target among gapped-circle distractors. The participants performed the task under high and low executive WM load, and the visual quality (Experiment 1) or discriminability of targets and distractors (Experiment 2) was manipulated across trials. By the logic of the additive factors method (Sternberg, 1969, 1998), if WM load compromises the quality of perceptual processing during visual search, manipulations of WM load and perceptual processing difficulty should produce nonadditive effects. Contrary to this prediction, the effects of WM load and perceptual difficulty were additive. The results imply that executive WM load does not degrade perceptual analysis during visual search.
\end{abstract}

Theoretical considerations and empirical evidence suggest that working memory (WM) plays a vital role in visual search (Awh, Vogel, \& Oh, 2006). The biased competition model (Desimone \& Duncan, 1995) and similar accounts of visual search (Bundesen 1990; Duncan \& Humphreys, 1989), for example, posit that an observer holds a target template in visual WM to guide attention while scanning. Consistent with this proposal, dual-task studies have shown that a visual WM load compromises the efficiency of visual search (Oh \& Kim, 2004; Woodman \& Luck, 2004; Woodman, Luck, \& Schall, 2007; but see Woodman, Vogel, \& Luck, 2001, for an exception) and that attention is often biased toward stimuli that match the contents of visual WM (Downing, 2000; Olivers, Meijer, \& Theeuwes, 2006; Soto, Heinke, Humphreys, \& Blanco, 2005; but see Downing \& Dodds, 2004; Houtkamp \& Roelfsema, 2006; Soto, Humphreys, \& Heinke, 2006; and Woodman \& Luck, 2007, for evidence of qualifications and exceptions). Perhaps more unexpectedly, search also appears to involve executive $\mathrm{WM}$, the component of the WM system also known to be responsible for such processes as coordinating multiple tasks (e.g., Baddeley, Chincotta, \& Adlam, 2001), generating novel behaviors (e.g., Baddeley, Emslie, Kolodny, \& Duncan, 1998), inhibiting prepotent responses (e.g., Unsworth, Schrock, \& Engle, 2004), and focusing and maintaining attention (e.g., Bleckley, Durso, Crutchfield, Engle, \& Khanna, 2003; Conway, Cowan, \& Bunting, 2001) (see Baddeley, 2007, and Engle, 2002, for reviews). Research has shown that the demand to maintain a search template can divert executive WM from a concurrent nonvisual task and that the amount of capacity consumed by the template is a function of target complexity (Bourke \& Duncan, 2005). Other work has demonstrated the converse pattern of interference, revealing that an executive loading task can reduce search efficiency (Anderson, Mannan, Rees, Sumner, \& Kennard, 2008; Han \& Kim, 2004) and suggesting again that visual search demands executive WM capacity. Studies of individual differences, moreover, have shown a correlation between WM capacity and performance in search tasks demanding high levels of top-down attentional control, as is needed to suppress salient distractors, for example (Sobel, Gerrie, Poole, \& Kane, 2007), or to maintain attentional focus on a subset of items within a field of distractors (Poole \& Kane, 2009).

A variety of data thus implicate executive WM as one of the cognitive mechanisms underlying visual search and suggest a number of roles that executive processes might play in search. To further explore the functions of executive WM in visual search, Peterson, Beck, and Wong (2008) examined the influence of executive load on searchers' eye movements. Participants performed a serial search task under single-task control conditions or while concurrently performing an executive WM task. Eye movement data revealed two changes to account for the observed increase in behavioral response times (RTs). First, the participants under high load were more likely to revisit an item that had already been fixated, particularly if the initial gaze on the item was very brief. Second, gaze durations for the items that did not receive a revisit were longer under high 
load. Peterson et al. inferred two mechanisms by which executive load hindered search. To account for the effect of executive load on revisitation rates, they posited that high load produced a tendency toward premature attention shifts, allowing the eyes to disengage from a fixated object before perceptual processing had completed and thus demanding a revisit. To account for the effect of executive load on gaze durations, Peterson et al. speculated that high load slowed visual processing, increasing the time needed for a fixated item to be identified.

Both of the mechanisms of interference posited by Peterson et al. (2008) are plausible and are consistent with evidence for a role of executive WM in controlling attention (e.g., Conway et al., 2001; Kane, Bleckley, Conway, \& Engle, 2001; Lavie, Hirst, de Fockert, \& Viding, 2004) and regulating stimulus access to conscious awareness (Fougnie \& Marois, 2007). The speculation that load impaired visual processing is furthermore consistent with the finding that prefrontal regions involved in executive WM (Kane \& Engle, 2002) serve to modulate extrastriate neuronal activity produced by visual stimulation (Barceló, Suwazono, \& Knight, 2000) and, more generally, with the finding that top-down attentional control modulates the quality of visual stimulus representation (e.g., Pessoa, Kastner, \& Ungerleider, 2003; Reynolds \& Chelazzi, 2004). Peterson et al.'s (2008) conclusion that visual processing during search was slowed by executive load, however, was premised largely on the finding that gaze durations for nonrevisited items were longer under high than under low load. Unfortunately, a change in gaze duration by itself reveals little about underlying perceptual or cognitive processes (Viviani, 1990). An increase in the time spent fixating an item, therefore, cannot be taken as firm evidence for impaired visual processing but might instead, for example, reflect changes in decisional or oculomotor processes. To determine whether perceptual processing in visual search is compromised by executive load, more direct measures of the influence of load on perceptual processing quality are necessary.

Toward this goal, in the present experiments, we manipulated the bottom-up difficulty of visual stimulus analysis and tested data for interactions of processing difficulty by load, interpreting the results within the framework of Sternberg's $(1969,1998)$ additive factors method (AFM). The AFM seeks to localize the influence of stimulus and task manipulations within the cognitive processing stream by identifying patterns of additive and nonadditive effects between independent variables in RTs (Sternberg, 1969, 1998) and log-transformed accuracy rates (Schweickert, 1985): Where two or more manipulations produce statistical interactions, they are inferred to influence at least one common stage of processing; where they produce additive effects, they are inferred to influence separate processing stages. Work using the AFM, notably, has implied that front-end visual processing is independent of higher level response selection and execution processes. For instance, Sternberg (1969) found that visual stimulus degradation slowed choice RTs in a pattern recognition task but that the effects of stimulus quality were additive with the effects of stimulus-response compatibility. From these results, he inferred a visual encoding stage susceptible to interference from stimulus degradation but independent of responserelated processes. Logan (1978), similarly, found additive effects of visual noise and set size in a visual search task and concluded that visual encoding constituted a stage of processing distinct from target-distractor comparison. A number of further studies, reviewed by Sanders (1998), showed corroborative data.

To gauge the effects of executive load on visual processing during search, we examined performance data for interactions of executive WM load with stimulus quality (Experiment 1) and target-distractor discriminability (Experiment 2). If executive load compromises perceptual processing, then the manipulation of load should interact with the manipulation of perceptual processing difficulty.

\section{EXPERIMENT 1}

\section{Method}

Participants. The participants were 24 young adults (6 male, mean age $=20.7$ years) with normal or corrected-to-normal visual acuity. Each participant received $\$ 8$ for taking part in the experiment.

Apparatus and Stimuli. Eye movements were recorded with a desktop-mounted EyeLink 1000 eyetracking system (SR Research, Mississauga, ON) with a spatial resolution of $0.5^{\circ}$ and a temporal resolution of $1000 \mathrm{~Hz}$. Stimuli were presented on a 19-in. ViewSonic E90FB CRT monitor with a resolution of $1,024 \times 768$ pixels and a vertical refresh rate of $75 \mathrm{~Hz}$. The stimuli were viewed from a distance of $77 \mathrm{~cm}$, held constant by a chinrest.

In the stimulus display, each trial consisted of one full circle target and 35 gapped-circle distractors, all drawn in black on a white background. On half of the trials, the stimuli were presented intact, with no degradation (Figure 1A). On the remaining trials, each item was degraded with 700 pixels of noise within a $59 \times 59$ pixel (Figure 1B) square centered on the item. To maintain a constant mean luminance of the individual items when the stimuli were degraded, 350 randomly chosen black pixels were turned to white, and 350 randomly chosen white pixels were turned to black.

The inner diameter of each item was $0.90^{\circ}$, and the outer diameter was $1.50^{\circ}$. The gap size of the distractors was $0.31^{\circ}$. The stimulus items were arranged to form six rows of six columns each, with the items in a row separated center-to-center by $3.66^{\circ}$. Alternating rows were offset by $1.83^{\circ}$ in opposite directions to create a hexagonal pattern. In half of the experimental blocks, all distractors were oriented to face toward the target in one of the four cardinal directions (after Hooge \& Erkelens, 1998). Within the other blocks of trials, the stimuli were created by first generating an image in which distractors were oriented to face the target, and then rotating all of the distractors by a common value of $90^{\circ}, 180^{\circ}$, or $270^{\circ}$, chosen randomly. The manipulation of distractor orientation was included as a test of hypotheses beyond those discussed here. However, the data showed no interactions of orientation and WM load. For the sake of clarity, therefore, the data are collapsed across distractor orientation for analysis, and distractor orientation is not discussed further.

Procedure. The participants performed concurrent visual search and WM tasks each trial. The search task required the participants to find and fixate a target - an ungapped circle among gapped-circle distractors. In low-WM load conditions, the participants were asked to count forward by ones from a random threedigit starting value, and in high-load conditions, they were asked to count backward by threes - a conventional executive loading task (e.g., Han \& Kim, 2004).

Each trial was preceded by a blank screen with a central fixation cross. The participants initiated a trial by pressing the space bar on the computer keyboard. The trial began with a 1,000-msec text mes- 
A

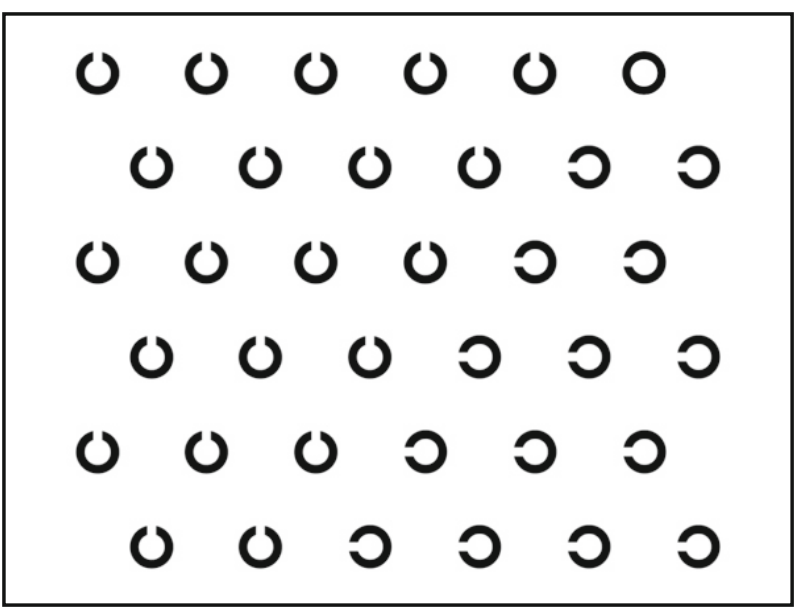

B

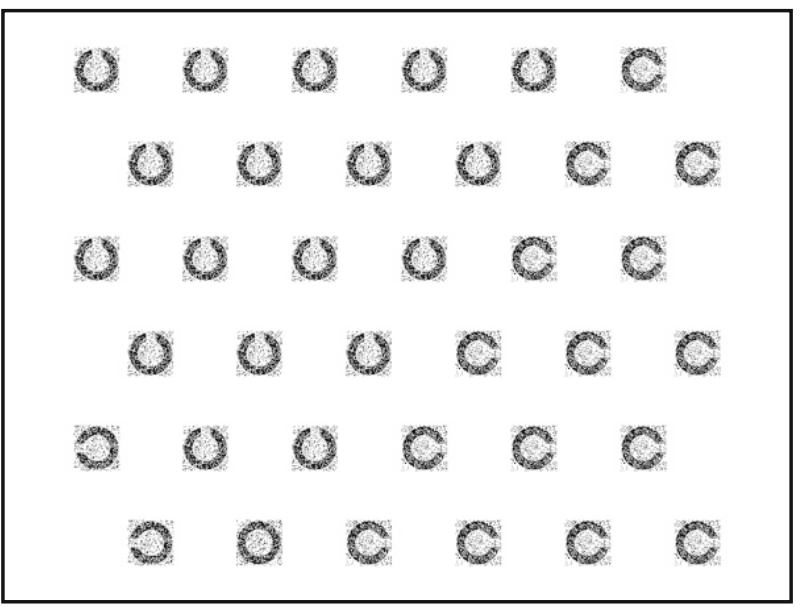

Figure 1. Example of undegraded (A) and degraded (B) stimulus displays of Experiment 1.

sage reminding the participants whether to count forward by ones or backward by threes, followed by a 1,000-msec display of the starting number for the trial. Next, a single circle appeared at 1 of the 36 possible target locations, chosen randomly. To discourage the use of stereotyped scanning strategies during search, the participants were required to fixate this circle before beginning the search task. Finally, $300 \mathrm{msec}$ after the participants' gaze landed on the circle, the search display appeared. The participants were instructed to find and fixate the target and then to press the space bar to end the search task. The trial timed out after $20 \mathrm{sec}$ without a manual response. The participants were asked to begin counting when the starting number appeared and to continue until terminating the search. At the end of a trial, the participants were prompted to report the number at which they had stopped counting by typing it on a numeric keypad.

The quality of the visual stimuli (undegraded vs. degraded) and the level of WM load imposed by the counting task (low vs. high) were manipulated factorially. An experimental session comprised four blocks of 76 trials each, two blocks each under high and low WM load. The order of high- and low-load blocks was counterbalanced across participants, and the participants were informed before the start of each block about which loading task to perform. Unde- graded and degraded stimulus trials were mixed within blocks. Each block began with 4 randomly chosen practice trials, 2 each using undegraded and degraded stimuli. The remaining 72 trials were experimental trials, half using undegraded and half using degraded stimuli. Within the experimental trials, the target stimulus appeared twice at each of the 36 different possible locations, once each with degraded and undegraded stimuli. The order of trials within a block was randomized.

\section{Results}

The data from 1 participant who failed to complete all of the conditions of the experiment and from 1 participant who fixated the target at the time of the manual response on fewer than $50 \%$ of the trials were excluded from analysis. For the remaining participants, the analysis excluded practice trials, trials on which the search task ended without a manual response, and trials on which the participants were not fixating the target at the time of the manual response. This produced a loss of 590 trials $(8.5 \%)$ across all conditions and participants, leaving 6,322 trials for analysis. The analyses of visual search data included trials on which an error occurred on the counting task. However, the analyses that excluded those trials produced patterns of effects identical to those reported below. For statistical analysis, the data were submitted to two-way withinsubjects ANOVAs, with stimulus quality (undegraded vs. degraded) and WM load (low vs. high) as factors.

RTs. Figure 2 presents mean RTs for visual search, measured as the time between onset of the search display and execution of the manual response. As was expected, reliable main effects indicated that RTs were longer with degraded stimuli than with undegraded stimuli $[F(1,21)=111.219$, $\left.p<.001, \eta_{\mathrm{p}}^{2}=.841\right]$ and were longer under high WM load than under low load $\left[F(1,21)=15.489, p=.001, \eta_{\mathrm{p}}^{2}=\right.$ $.424]$. These results provide a check that both experimental manipulations were successful. The analysis showed no interaction of WM load with stimulus quality $[F(1,21)=$ $\left.0.956, p=.339, \eta_{\mathrm{p}}^{2}=.044\right]$, however - a result that, by the reasoning of the AFM, implies that WM load and stimulus quality did not influence a common processing stage.

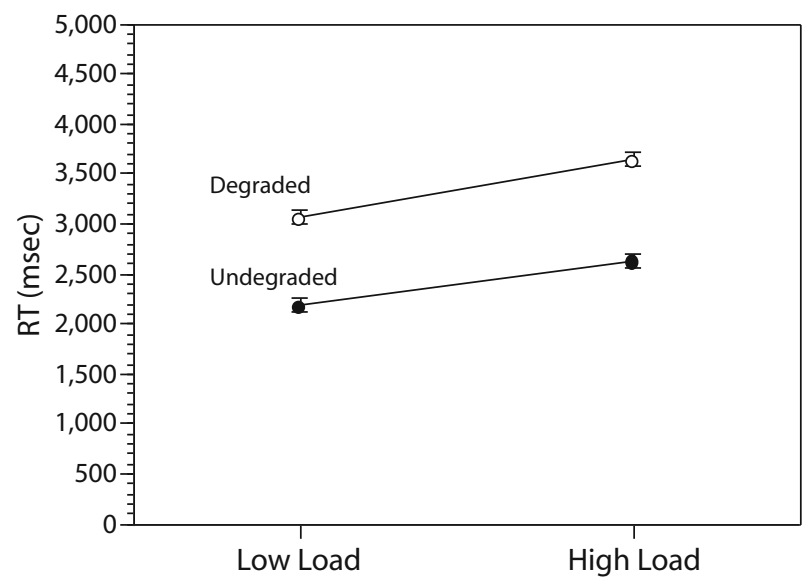

Figure 2. Mean manual response times (RTs) in Experiment 1. Error bars in all figures indicate within-subjects standard errors (Loftus \& Masson, 1994) based on the interaction term. 


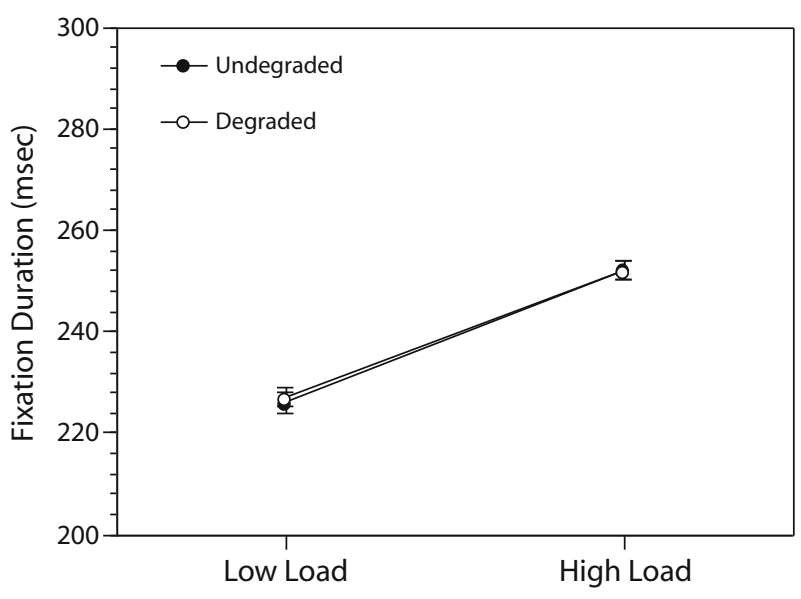

Figure 3. Mean fixation durations in Experiment 1.

Fixation frequency and fixation duration. Oculomotor data were analyzed to determine the bases of the WM and stimulus degradation effects in the RT data. The mean number of fixations made between onset of the search display and execution of the manual response on each trial ranged across conditions from a low of 8.35 to a high of 12.79 and was correlated almost perfectly $(r=$ .97) with mean RT. Accordingly, the pattern of statistical effects in the fixation frequency data was identical to that for the RT data. Notably, however, the raw effect size of the influence of executive WM load was small; on average, high WM load increased the number of fixations per trial by less than $1.0(M=0.88)$, suggesting that the larger influence of load on search times arose from changes in fixation durations.

A preliminary analysis of fixation durations indicated that mean durations changed systematically across the course of a trial (cf. Antes, 1974; Unema, Pannasch, Joos, \& Velichkovsky, 2005). Because of this, an analysis collapsing fixation durations over the full course of each trial would confound the effects of the experimental manipulations with the effects of the number of fixations made per trial. Our statistical analysis therefore included only the first six fixations of each trial, after which empty data cells began to occur for some participants in some experimental conditions. The preliminary analysis indicated no interactions between ordinal fixation position (first, second, etc.) with either stimulus quality or WM load. Ordinal fixation position was therefore not included as a factor in the analysis reported here. The analysis also excluded the last gaze of every trial, which included the time needed for manual response execution, and excluded fixations briefer than $100 \mathrm{msec}$ ( $8 \%$ of fixations for the low-load conditions, $7 \%$ for the high-load conditions).

Mean fixation durations are presented in Figure 3. The analysis indicated that fixations were longer under high executive load than under low load $[F(1,21)=17.514$, $\left.p<.001, \eta_{\mathrm{p}}^{2}=.455\right]$ but, surprisingly, showed no reliable main effect of stimulus quality $[F(1,21)=0.013, p=.909$, $\left.\eta_{\mathrm{p}}^{2}=.001\right]$ and, moreover, showed no hint of an interaction between WM load and stimulus quality $[F(1,21)=$ $\left.0.331, p=.571, \eta_{\mathrm{p}}^{2}=.016\right]$. This pattern of effects was unchanged when fixations of less than $100 \mathrm{msec}$ were included in the analysis.

Percentage of saccades toward the target. As a further check of the influence of executive load on search performance, we examined the percentage of saccades that was made each trial in the direction of the target. In effect, we treated each saccade as a discrete response and classified those toward the target as correct responses. A higher percentage of correctly directed saccades indicates a more efficient search, with the eyes being guided more effectively toward the target (Wolfe, 1994, 1998; Wolfe, Cave, \& Franzel, 1989). For the analysis, a saccade was classified as being made toward the target if its direction was within $\pm 45^{\circ}$ of the target's direction relative to the saccade launch point; analyses using criteria more or less restrictive than this produced similar results. Mean raw values are shown in Figure 4. For the statistical analysis, the data were log transformed; Schweikert (1985) showed that manipulations influencing separate processing stages exert additive influences on log-transformed correct response rates. A reliable main effect of stimulus quality indicated that the frequency of saccades in the target direction was higher when the stimuli were undegraded than when they were degraded $[F(1,21)=53.530 p<.001$, $\left.\eta_{\mathrm{p}}^{2}=.718\right]$ and a reliable main effect of executive load $\left[F(1,21)=5.977, p=.023, \eta_{\mathrm{p}}^{2}=.222\right]$ indicated that high load decreased the proportion of saccades in the correct direction. The effects of stimulus quality and executive load, however, were additive $[F(1,21)=0.856, p=$ $\left..365, \eta_{\mathrm{p}}^{2}=.039\right]$. An analysis of the nontransformed data produced similar effects. An analysis of fixation durations indicated that presaccadic fixations were briefer preceding a movement in the incorrect direction $(M=227 \mathrm{msec})$ than preceding a movement in the direction of the target $(M=244 \mathrm{msec})\left[F(1,21)=9.369, p=.006, \eta_{\mathrm{p}}^{2}=.309\right]$, but this effect was not modulated by any interactions with executive load or stimulus quality (all $p$ s $>.10$ ).

Target recognition failures. For a converging measure of perceptual processing quality, we examined the

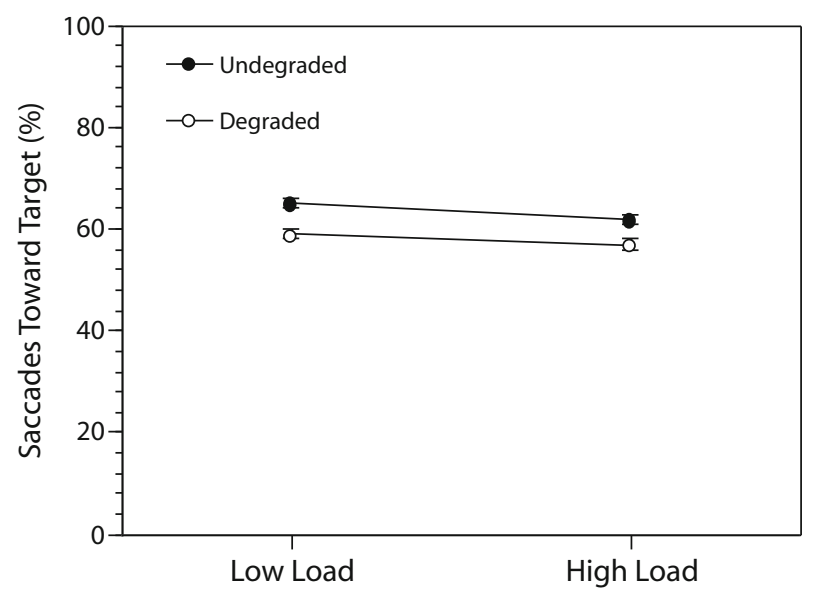

Figure 4. Mean percentage of saccades made in the direction of the target in Experiment 1. 
frequency of target recognition failures-instances in which the participants fixated the target object but then continued to search without executing a manual response. A fixation was classified as being on the target if it fell closer to the target object than to any of the distractors. The proportion of target recognition failures was defined as the number of recognition failures divided by the total number of target fixations. The data were again log transformed for statistical analysis. The data values reported in the text are nontransformed. The results indicated that the percentage of target recognition failures trended toward being lower under low executive load $(M=30.3 \%)$ than under high load $(M=35.1 \%)[F(1,21)=2.831, p=$ $\left..107, \eta_{\mathrm{p}}^{2}=.119\right]$ and was lower when stimuli were intact than when they were degraded $(M=18 \%$ vs. $47.0 \%)$ $\left[F(1,21)=150.894, p<.001, \eta_{\mathrm{p}}^{2}=.878\right]$, but showed no hint of an interaction $\left[F(1,21)=.105, p=.749, \eta_{\mathrm{p}}^{2}=\right.$ $.005]$. An analysis of the nontransformed data also failed to indicate an interaction.

Loading task performance. With a final pair of analyses, we examined counting accuracy and speed to ensure that the participants did not sacrifice loading task performance to protect visual search in the high-load degraded stimulus conditions. A counting error was defined as any instance in which the number reported at the end of the trial could not have been reached by counting as instructed from that trial's starting number. For the lowload condition, this included trials on which the reported number was less than or equal to the starting number. For the high-load conditions, this included trials on which the difference between the reported number and the starting number was not a negative integer multiple of three. The mean error rate was smaller in the low-load than in the high-load conditions $(M=4.2 \%$ vs. $17.4 \%)[F(1,21)=$ $32.989, p<.001, \eta_{\mathrm{p}}^{2}=.611$ ] but did not differ reliably as a function of stimulus quality $(M=11.3 \%$ for intact stimuli vs. $10.4 \%$ for degraded stimuli) $[F(1,21)=1.106, p=$ $\left..305, \eta_{\mathrm{p}}^{2}=.050\right]$ and showed no interaction of load and stimulus quality $\left[F(1,21)=0.791, p=.384, \eta_{\mathrm{p}}^{2}=.036\right]$. Counting rate, defined as the number of steps counted on each trial divided by the RT, served as a second measure of loading task performance. This rate was higher in low-load than in high-load conditions $(M=1.70 \mathrm{vs}$. $0.98 \mathrm{steps} / \mathrm{sec})\left[F(1,22)=10.772, p=.004, \eta_{\mathrm{p}}^{2}=.339\right]$, as was expected, and was modestly higher when the stimuli were undegraded than when they were degraded $(M=$ 1.39 vs. $1.29 \mathrm{steps} / \mathrm{sec})\left[F(1,21)=8.556, p=.008, \eta_{\mathrm{p}}^{2}=\right.$ .289]. The data showed no interaction of load and search $\operatorname{task}\left[F(1,21)=0.002, p=.969, \eta_{\mathrm{p}}^{2}<.001\right]$, however.

\section{Discussion}

Consistent with earlier work (Anderson et al., 2008; Han \& Kim, 2004; Peterson et al., 2008), in Experiment 1, there were significant and substantial costs of executive WM load for visual search performance, evident in RTs, fixation frequencies, fixation durations, and a measure of saccadic guidance. The data likewise confirmed that search was hindered by stimulus degradation, a manipulation known to compromise perceptual processing quality. By every measure of performance examined, however, the effects of executive load and stimulus quality were additive. The results confirm that executive load slows search but offer no evidence that it does so by compromising perceptual processing. In Experiment 2, we sought converging evidence for this conclusion.

\section{EXPERIMENT 2}

In Experiment 1, we found no evidence of an interaction of stimulus quality and executive load, where stimulus quality was manipulated by the addition of visual noise. In Experiment 2, we aimed to extend the results of Experiment 1 by using an alternative manipulation of visual processing difficulty. Here, the size of the gap in the gapped-circle distractors was varied to manipulate targetdistractor discriminability. Like stimulus quality, stimulus discriminability appears to influence visual processing, independent of higher level decision and response selection processes (Sanders, 1998).

\section{Method}

Participants. The participants were 14 young adults (6 male, mean age $=23.93$ years) with normal or corrected-to-normal visual acuity. Each participant received $\$ 8$ for taking part in the experiment.

Stimulus, Apparatus, and Procedure. The apparatus was identical to that of Experiment 1. The stimuli and procedure were identical to those of Experiment 1, except that the noise-degraded stimuli were replaced with undegraded stimuli whose distractor gap size was $0.08^{\circ}$. The gap size for the high-discriminability stimuli was again $0.31^{\circ}$.

\section{Results}

The data from 3 participants were excluded from the analysis because those participants were fixating the target item at the time of their manual response on fewer than $50 \%$ of all trials. The treatment of the remaining data was identical to that of Experiment 1. Data filtering produced a loss of 130 trials $(4.10 \%)$ across all conditions and participants, leaving 3,038 trials for analysis. Analyses of visual search data that excluded trials with counting errors produced patterns of effects identical to those reported below. For the statistical analysis, the data were submitted to two-way within-subjects ANOVAs with targetdistractor discriminability and WM load as factors.

RTs. Figure 5 presents mean RTs. RTs increased when target-distractor discriminability decreased $[F(1,10)=$ $\left.83.514, p<.001, \eta_{\mathrm{p}}^{2}=.893\right]$ and were longer under high WM load than under low load $[F(1,10)=7.520, p=.021$, $\left.\eta_{\mathrm{p}}^{2}=.429\right]$ but showed no interaction $[F(1,11)<0.001$, $\left.p=.987, \eta_{\mathrm{p}}^{2}<.001\right]$.

Fixation frequency and fixation duration. The mean number of fixations made on each trial ranged across conditions from 9.20 to 13.84 and was again highly correlated across conditions with mean RT $(r=.96)$. As in Experiment 1, the mean number of fixations per trial was modestly higher under high-load than under low-load conditions (mean difference $=0.98$ ). Here, however, this difference was not reliable. The statistical analysis produced only a main effect of target-distractor discriminability $\left[F(1,10)=96.716, p<.001, \eta_{\mathrm{p}}^{2}=.906\right]$, showing nei- 


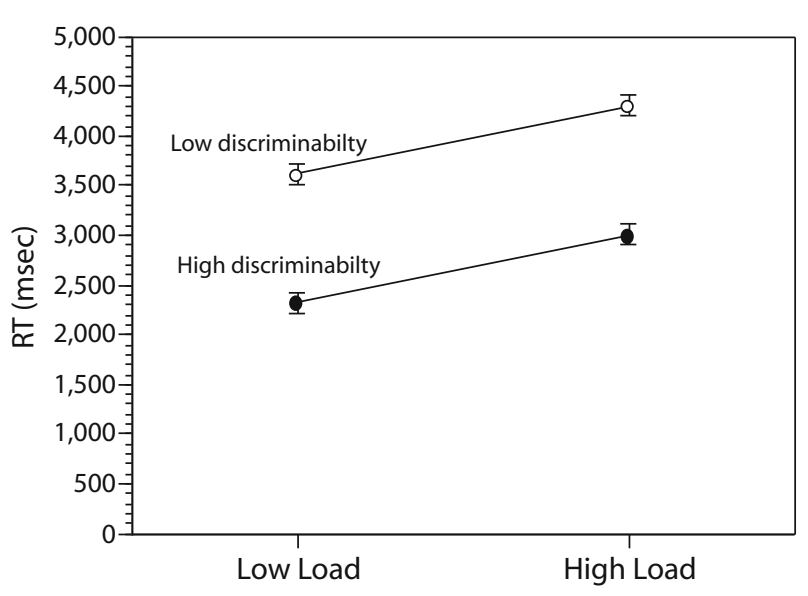

Figure 5. Mean manual response times (RTs) in Experiment 2.

ther a reliable main effect of WM load $[F(1,10)=1.090$, $\left.p=.321, \eta_{\mathrm{p}}^{2}=.098\right]$ nor a reliable interaction $[F(1,10)=$ $\left.0.490, p=.400, \eta_{\mathrm{p}}^{2}=.047\right]$.

The analysis of fixation durations included only the first five fixations of each trial, after which empty data cells began to occur for some participants. As in Experiment 1, the analysis excluded the last gaze of every trial, along with fixations briefer than $100 \mathrm{msec}$ ( $6 \%$ of fixations for low-load conditions, $5 \%$ for high-load conditions). Mean fixation durations are presented in Figure 6. The fixation durations were again longer under high WM load than under low load $\left[F(1,10)=15.375, p=.003, \eta_{\mathrm{p}}^{2}=.606\right]$ but showed no reliable main effect of target-distractor discriminability $\left[F(1,10)=.886, p=.369, \eta_{\mathrm{p}}^{2}=.081\right]$ and no interaction $\left[F(1,10)=0.112, p=.745, \eta_{\mathrm{p}}^{2}=.011\right]$. This pattern of effects was unchanged when fixations of less than $100 \mathrm{msec}$ were included in the analysis.

Percentage of saccades toward target. The mean raw values of the percentage of saccades made toward the target on each trial are shown in Figure 7. A statistical analysis of the log-transformed proportion correct data indicated that the relative frequency of saccades in the target direction was higher when the target and distractors were highly discriminable than when they were not $[F(1,10)=$ 74.887, $\left.p<.001, \eta_{\mathrm{p}}^{2}=.882\right]$ but showed no reliable main effect of executive load $\left[F(1,10)=0.509, p=.492, \eta_{\mathrm{p}}^{2}=\right.$ $.048]$ and no interaction $\left[F(1,10)=0.226, p=.645, \eta_{\mathrm{p}}^{2}=\right.$ $.022]$. An analysis of the nontransformed data produced similar effects. The presaccadic fixation duration did not differ reliably between saccades made in the incorrect direction $(M=245 \mathrm{msec})$ and those made in the correct direction $(M=253 \mathrm{msec})\left[F(1,21)=2.244, p=.165, \eta_{\mathrm{p}}^{2}=\right.$ $.183]$ and showed no interaction of saccade direction with executive load or stimulus quality (all $p$ s $>.10$ ).

Target recognition failures. The percentage of target recognition failures was lower when the targets and distractors were highly discriminable than when they were not ( $M=8.1 \%$ vs. $18.3 \%$, respectively) $[F(1,10)=$ $\left.71.964, p<.001, \eta_{\mathrm{p}}^{2}=.878\right]$ but showed no reliable main effect of WM load $(M=13 \%$ for low-load conditions vs. $13.5 \%$ for high-load conditions) $[F(1,10)=0.298, p=$ $\left..597, \eta_{\mathrm{p}}^{2}=.029\right]$, and no hint of an interaction $[F(1,10)=$ $\left.0.200, p=.664, \eta_{\mathrm{p}}^{2}=.020\right]$. An analysis of the nontransformed data produced similar effects.

Loading task performance. The mean error rate on the loading task was higher in the high-executive-load conditions than in low-load conditions ( $M=16 \%$ vs. $5 \%$ ) $\left[F(1,10)=7.608, p=.020, \eta_{\mathrm{p}}^{2}=.432\right]$ but did not differ reliably as a function of target-distractor discriminability ( $M=9.8 \%$ for high-discriminability stimuli vs. $11.3 \%$ for low-discriminability stimuli) $[F(1,10)=2.037, p=.184$, $\left.\eta_{\mathrm{p}}^{2}=.169\right]$ and showed no interaction $[F(1,10)=0.218$, $\left.p=.650, \eta_{\mathrm{p}}^{2}=.021\right]$. The counting rate was higher in the low-load than in the high-load conditions $(M=1.68$ vs. $0.98 \mathrm{steps} / \mathrm{sec})\left[F(1,10)=22.902, p=.001, \eta_{\mathrm{p}}^{2}=\right.$ .696] and was higher when the targets and distractors were highly discriminable ( $M=1.39$ vs. $1.28 \mathrm{steps} / \mathrm{sec})$ $\left[F(1,10)=17.046, p=.002, \eta_{\mathrm{p}}^{2}=.630\right]$ but also showed no interaction $\left[F(1,10)=0.001, p=.981, \eta_{\mathrm{p}}^{2}<.001\right]$.

\section{Discussion}

The results closely parallel those of Experiment 1: Although manipulations of executive WM load and targetdistractor discriminability both had a substantial influence on visual search performance, their effects were strictly additive across an array of performance measures. The data again gave no evidence that executive load compromised visual analysis.

\section{GENERAL DISCUSSION}

In two experiments, we found large and reliable effects of an executive loading task on visual search performance, evident in performance measures including RTs, fixation frequencies, fixation durations, and the percentage of saccades made toward the target object. In no case, however, did the influence of executive WM load interact with that of perceptual processing difficulty. Across both experiments and all dependent measures examined, rather, the effects of executive load and perceptual processing difficulty were additive. By the reasoning of the

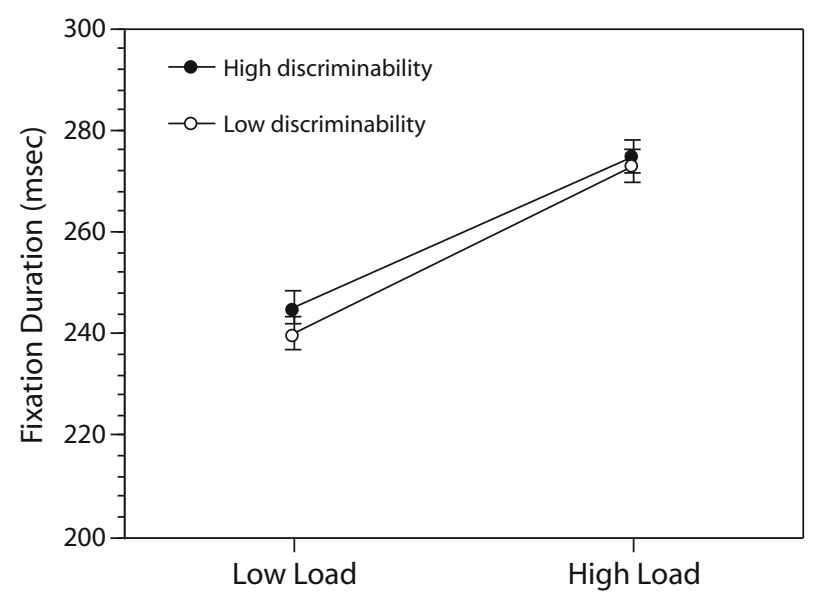

Figure 6. Mean fixation durations in Experiment 2. 


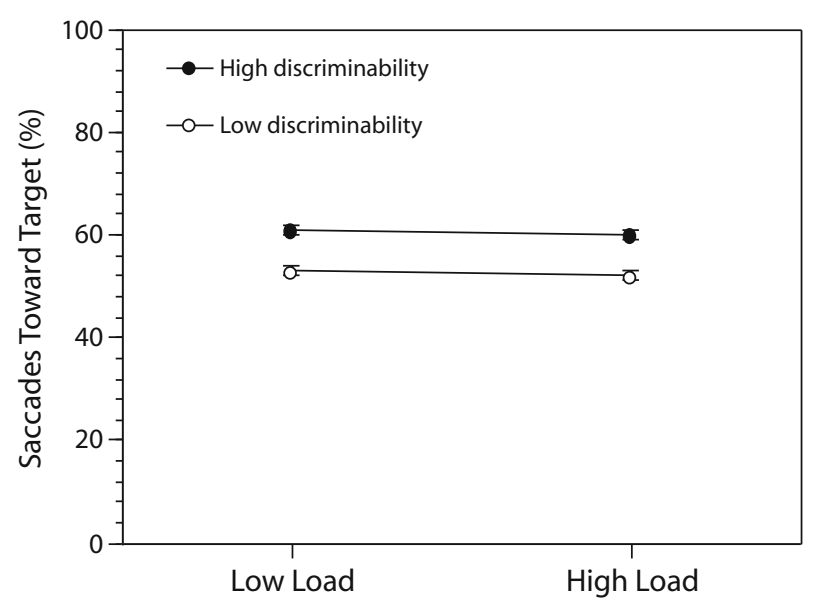

Figure 7. Mean percentage of saccades made in the direction of the target in Experiment 2.

AFM, such effects imply that changes of executive load and manipulations of perceptual difficulty affect search in fundamentally different ways. Contrary to earlier conclusions, executive load does not appear to hinder perceptual processing.

Is it possible that the inference of additivity reflects a simple failure to detect a true interaction between executive load and perceptual processing difficulty? Although the possibility of a Type II statistical error is necessarily a concern when applying the AFM, several considerations argue against that worry here. First, additive effects were obtained in two separate experiments, using two different manipulations of perceptual processing difficulty. Second, additivity prevailed across a variety of dependent measures, including measures of processing speed and accuracy, and measures at the levels of both behavioral responses and individual saccades. Third, $p$ values for the interactions of executive load and perceptual processing uniformly failed to even approach statistical significance. Finally, the conclusion that visual processing is unaffected by executive load is consistent with an array of existing data. As was noted above, for example, Sternberg (1969; see also Sanders, 1998) reported that the effects of visual stimulus quality on RTs are additive with those of response selection difficulty, typically considered a form of executive processing demand. Similarly, Pashler (1984) reported additive effects of visual stimulus contrast and dual-task interference in experiments using a visual search task within the psychological refractory period (PRP) paradigm, whereas Logan (1978) described additive influences of visual stimulus quality and short-term memory load on performance in a search task. All of these results imply that front-end visual processing is largely immune to interference from central cognitive processing load (Pashler, 1994).

How, then, does executive load hinder search? One possibility, as was suggested by Peterson et al. (2008), is that high executive load triggers premature disengagements of attention, allowing the eyes to leave an attended object before normal presaccadic processing has been completed and thereby increasing the number of saccades and fixations needed to eventually locate the target. Such an effect would comport with the finding of the present Experiment 1 that the percentage of saccades made in the direction of the target was smaller under high load than under low load; given that correctly directed movements required longer presaccadic fixation durations than incorrectly directed movements, premature attentional disengagements would presumably increase the frequency of misdirected saccades. The premature disengagement hypothesis would also explain, at least in part, the finding here that more fixations were required to locate a target under high load than under low load. However, two pieces of evidence in the present data suggest that if high load engendered premature eye movements, the size of this effect was modest at best. First, the difference in the mean number of fixations between the high- and low-load conditions was less than 1 per trial (an effect that was nonsignificant in Experiment 2 ), accounting for only a very modest increase in behavioral RTs. Second, mean fixation durations were longer under high load than under low load. A tendency toward premature attention shifts, by itself, would obviously produce the opposite effect. An alternative mechanism of interference is needed to explain the increase in fixation durations observed under high executive load.

Thus, neither the perceptual processing hypothesis nor the premature attentional engagement hypothesis seems compatible with the present results. At least two alternative possibilities, however, seem consistent with the present findings and with an assortment of earlier data. A hypothesis related to the premature disengagement account but perhaps more plausible in light of the present findings is that executive load hinders postperceptual mechanisms responsible for guiding search (Wolfe, 1994; Wolfe et al., 1989), by either disrupting distractor suppression (Poole \& Kane, 2009; Sobel et al., 2007) or compromising the searcher's ability to maintain a target template (Bourke \& Duncan, 2005). By this speculation, increased fixation durations would be necessary under high load to compensate for poor attentional guidance, and a decreased tendency to saccade in the direction of the target, producing an increase in the number of saccades needed to locate the target, would reflect occasional failures of this compensatory process. Pop-out search would presumably be immune to executive load (Anderson et al., 2008; Han \& Kim, 2004), simply because high target salience would allow rapid detection even if attentional guidance was imperfect.

Another parsimonious account of the present findings might be found in the models of central task postponement that have been offered to explain the PRP. A wide variety of data indicate that the delays occurring when two tasks are performed in close succession are the result of a bottleneck in central cognitive processing, most specifically in response selection (Luck, 1998; McCann \& Johnston, 1992; Pashler, 1984, 1994; Pashler \& Johnston, 1989). As response selection is ongoing for one task, response selection for the second task is postponed. When punctate tasks are performed in sequence at short stimulus onset asynchronies, the consequence of this postponement is that responses for the second task are delayed, resulting 
in a classical PRP effect. When concurrent tasks are performed over time, as in the present experiments, the consequence is that access to response selection mechanisms is interleaved, with processing for one task or the other being intermittently postponed as the other task occupies the bottleneck (Pashler, 1998). In the present experiments, this implies that processing on the visual search task would be periodically suspended as the bottleneck was occupied by the loading task.

The postponement hypothesis assumes, however, that visual search would require recurring access to a response selection bottleneck. Why would this be so, if the participants' only explicit response demand was an end-oftrial keypress? One potential reason is that the decision to classify an attended item as a target or distractor demands bottleneck access, although this speculation may be undermined by Logan's (1978) finding that short-term memory load does not hinder target-distractor comparisons. An alternative possibility is that voluntary eye movement generation is bottleneck limited, meaning that an executive loading task would interfere with the saccade programming necessary to carry out an oculomotor search. Several pieces of evidence support this speculation. Pashler, Carrier, and Hoffman (1993), for example, reported that an auditory judgment can delay voluntary saccade execution in a PRP paradigm, and an experiment by Stuyven, van der Goten, Vandierendonck, Claeys, and Crevits (2000) demonstrated that executive load can increase voluntary saccade latencies even in a simple cued eye movement task. Other work has shown that WM capacity, hypothesized to be a reflection of executive processing (Engle, 2002), predicts movement latency in an endogenously cued saccade task (Unsworth et al., 2004). Under the saccade postponement hypothesis, finally, popout search (Anderson et al., 2008; Han \& Kim, 2004) and unspeeded search of very brief displays ${ }^{1}$ (Pashler, 1989; but see De Jong \& Sweet, 1994, for qualification) would be immune to executive load, because neither would require access to saccade programming. In total, these considerations suggest the process of saccade programming as a potentially important locus of interaction between executive control and visual search.

\section{AUTHOR NOTE}

We thank Jeremy Wolfe, Rozemarijn Houtkamp, and Kenith Sobel for helpful comments on an earlier draft of the article. Correspondence concerning this article should be addressed to J. S. McCarley, Beckman Institute, University of Illinois, 405 N. Matthews, Urbana, IL 61801 (e-mail: mccarley@illinois.edu).

\section{REFERENCES}

Anderson, E. J., Mannan, S. K., Rees, G., Sumner, P., \& Kennard, C. (2008). A role for spatial and nonspatial working memory processes in visual search. Experimental Psychology, 55, 301-312.

ANTES, J. R. (1974). The time course of picture viewing. Journal of Experimental Psychology, 103, 62-70.

AwH, E., Vogel, E. K., \& OH, S.-H. (2006). Interactions between attention and working memory. Neuroscience, 139, 201-208.

BADDELEY, A. D. (2007). Working memory, thought, and action. Oxford: Oxford University Press.

Baddeley, A. D., Chincotta, D., \& Adlam, A. (2001). Working mem- ory and the control of action: Evidence from task switching. Journal of Experimental Psychology: General, 130, 641-657.

Baddeley, A. D., Emslie, H., Kolodny, J., \& Duncan, J. (1998). Random generation and the executive control of working memory. Quarterly Journal of Experimental Psychology, 51A, 819-852.

Barceló, F., Suwazono, S., \& KNight, R. T. (2000). Prefrontal modulation of visual processing in humans. Nature Neuroscience, 3, 399-403.

Bleckley, M. K., Durso, F. T., Crutchfield, J. M., Engle, R. W., \& Khanna, M. M. (2003). Individual differences in working memory capacity predict visual attention allocation. Psychonomic Bulletin \& Review, 10, 884-889.

Bourke, P. A., \& DunCan, J. (2005). Effect of template complexity on visual search and dual-task performance. Psychological Science, 16, 208-213.

Bundesen, C. (1990). A theory of visual attention. Psychological Review, 97, 523-547.

Conway, A. R. A., Cowan, N., \& Bunting, M. F. (2001). The cocktail party phenomenon revisited: The importance of working memory capacity. Psychonomic Bulletin \& Review, 8, 331-335.

De JoNG, R., \& SweEt, J. B. (1994). Preparatory strategies in overlappingtask performance. Perception \& Psychophysics, 55, 142-151.

Dell'ACQua, R., \& Joliceur, P. (2000). Visual encoding of patterns is subject to dual-task interference. Memory \& Cognition, 28, 184-191.

Desimone, R., \& Duncan, J. (1995). Neural mechanisms of selective visual attention. Annual Review of Neuroscience, 18, 193-222.

DownING, P. E. (2000). Interactions between visual working memory and selective attention. Psychological Science, 11, 467-473.

Downing, P. E., \& DodDs, C. M. (2004). Competition in visual working memory for control of search. Visual Cognition, 11, 689-703.

Duncan, J., \& Humphreys, G. W. (1989). Visual search and stimulus similarity. Psychological Review, 96, 433-458.

ENGLE, R. W. (2002). Working memory capacity as executive attention. Current Directions in Psychological Science, 11, 19-23.

Fougnie, D., \& Marois, R. (2007). Executive working memory load induces inattentional blindness. Psychonomic Bulletin \& Review, 14, 142-147.

HaN, S. H., \& KIM, M. S. (2004). Visual search does not remain efficient when executive working memory is working. Psychological Science, 15, 623-628

Hooge, I. T. C., \& Erkelens, C. J. (1998). Adjustment of fixation duration in visual search. Vision Research, 38, 1295-1302.

HoutKamp, R., \& Roelfsema, P. R. (2006). The effect of items in working memory on the deployment of attention and the eyes during visual search. Journal of Experimental Psychology: Human Perception \& Performance, 32, 423-442.

JOLICEUR, P. (1999). Dual-task interference and visual encoding. Journal of Experimental Psychology: Human Perception \& Performance, 25, 596-616.

Joliceur, P., \& Dell'AcQua, R. (1998). The demonstration of shortterm consolidation. Cognitive Psychology, 36, 138-202.

Joliceur, P., \& Dell'ACQua, R. (1999). Attentional and structural constraints on visual encoding. Psychological Research, 62, 154-164.

Kane, M. J., Bleckley, M. K., Conway, A. R. A., \& Engle, R. W. (2001). A controlled-attention view of WM capacity. Journal of Experimental Psychology: General, 130, 169-183.

KANE, M. J., \& Engle, R. W. (2002). The role of prefrontal cortex in working memory capacity, executive attention, and general fluid intelligence: An individual-differences perspective. Psychonomic Bulletin \& Review, 9, 637-671

Lavie, N., Hirst, A., de Fockert, J. W., \& Viding, E. (2004). Load theory of selective attention and cognitive control. Journal of Experimental Psychology: General, 133, 339-354.

Loftus, G. R., \& MAsson, M. E. J. (1994). Using confidence intervals in within-subjects designs. Psychonomic Bulletin \& Review, 1, 476-490.

LOGAN, G. D. (1978). Attention in character-classification tasks: Evidence for the automaticity of component stages. Journal of Experimental Psychology: General, 107, 32-63.

LuCK, S. J. (1998). Sources of dual-task interference: Evidence from human electrophysiology. Psychological Science, 9, 223-227.

McCann, R. S., \& Johnston, J. C. (1992). Locus of the single-channel bottleneck in dual-task interference. Journal of Experimental Psychology: Human Perception \& Performance, 18, 471-484. 
Он, S.-H., \& Кıм, M.-S. (2004). The role of spatial working memory in visual search efficiency. Psychonomic Bulletin \& Review, 11, 275-281.

Olivers, C. N. L., Meijer, F., \& Theeuwes, J. (2006). Feature-based memory-driven attentional capture: Visual working memory content affects visual attention. Journal of Experimental Psychology: Human Perception \& Performance, 32, 1243-1265.

PASHLER, H. E. (1984). Processing stages in overlapping tasks: Evidence for a central bottleneck. Journal of Experimental Psychology: Human Perception \& Performance, 10, 358-377.

PASHLER, H. E. (1989). Dissociations and dependencies between speed and accuracy: Evidence for a two-component theory of divided attention in simple tasks. Cognitive Psychology, 21, 469-514.

PASHLER, H. E. (1994). Dual-task interference in simple tasks: Data and theory. Psychological Bulletin, 116, 220-244.

PASHLER, H. E. (1998). The psychology of attention. Cambridge, MA: MIT Press.

Pashler, H. E., Carrier, M., \& Hoffman, J. (1993). Saccadic eye movements and dual-task interference. Quarterly Journal of Experimental Psychology, 46A, 51-82.

Pashler, H. E., \& Johnston, J. C. (1989). Interference between temporally overlapping tasks: Chronometric evidence for central postponement with or without response grouping. Quarterly Journal of Experimental Psychology, 41A, 19-45.

Pessoa, L., Kastner, S., \& Ungerleider, L. G. (2003). Neuroimaging studies of attention: From modulation of sensory processing to topdown control. Journal of Neuroscience, 23, 3990-3998.

Peterson, M. S., Beck, M. R., \& Wong, J. H. (2008). Were you paying attention to where you looked? The role of executive working memory in visual search. Psychonomic Bulletin \& Review, 15, 372-377.

Poole, B. J., \& Kane, M. J. (2009). Working memory capacity predicts the executive control of visual search among distractors: The influences of sustained and selective attention. Quarterly Journal of Experimental Psychology, 62, 1430-1454.

Reynolds, J. H., \& Chelazzi, L. (2004). Attentional modulation of visual processing. Annual Review of Neuroscience, 27, 611-647.

SANDERS, A. F. (1998). Elements of human performance: Reaction processes and attention in human skill. Mahwah, NJ: Erlbaum.

SCHWEICKERT, R. (1985). Separable effects of factors on speed and accuracy: Memory scanning, lexical decision, and choice tasks. Psychological Bulletin, 97, 530-546.

Sobel, K. V., Gerrie, M. P., Poole, B. J., \& Kane, M. J. (2007). Individual differences in working memory capacity and visual search: The roles of top-down and bottom-up processing. Psychonomic Bulletin \& Review, 14, 840-845.

Soto, D., Heinke, D., Humphreys, G. W., \& Blanco, M. J. (2005). Early, involuntary top-down guidance of attention from working memory. Journal of Experimental Psychology: Human Perception \& Performance, 31, 248-261.

Soto, D., Humphreys, G. W., \& HeinKe, D. (2006). Dividing the mind: The necessary role of frontal lobes in separating memory from search. Neuropsychologia, 44, 1282-1289.

STERnBERG, S. (1969). The discovery of processing stages: Extensions of Donders' method. Acta Psychologica, 30, 276-315.
Sternberg, S. (1998). Discovering mental processing stages: The method of additive factors. In D. Scarborough \& S. Sternberg (Eds.), Methods, models, and conceptual issues: An invitation to cognitive science (Vol. 4, pp. 703-863). Cambridge, MA: MIT Press.

Stuyven, E., van der Goten, K., Vandierendonck, A., Claeys, K., $\&$ Crevits, L. (2000). The effect of cognitive load on saccadic eye movements. Acta Psychologica, 104, 69-85.

Unema, P. J. A., Pannasch, S., Joos, M., \& Velichkovsky, B. M. (2005). Time course of information processing during scene perception: The relationship between saccade amplitude and fixation duration. Visual Cognition, 12, 473-494.

Unsworth, N., Schrock, J. C., \& ENGLE, R. W. (2004). Working memory capacity and the antisaccade task: Individual differences in voluntary saccade control. Journal of Experimental Psychology: Learning, Memory, \& Cognition, 30, 1302-1321.

Viviani, P. (1990). Eye movements in visual search: Cognitive, perceptual, and motor control aspects. Reviews of Oculomotor Research, $\mathbf{4}$, 353-393.

WoLfE, J. M. (1994). Guided search 2.0: A revised model of visual search. Psychonomic Bulletin \& Review, 1, 202-238.

Wolfe, J. M. (1998). Visual search. In H. E. Pashler (Ed.), Attention (pp. 13-73). East Sussex: Psychology Press.

Wolfe, J. M., Cave, K. R., \& Franzel, S. L. (1989). Guided search: An alternative to the feature integration model for visual search. Journal of Experimental Psychology: Human Perception \& Performance, 15, 419-433.

Woodman, G. F., \& Luck, S. J. (2004). Visual search is slowed when visuospatial working memory is occupied. Psychonomic Bulletin \& Review, 11, 269-274.

Woodman, G. F., \& Luck, S. J. (2007). Do the contents of visual working memory automatically influence attentional selection during visual search? Journal of Experimental Psychology: Human Perception \& Performance, 33, 363-377.

Woodman, G. F., LucK, S. J., \& Schall, J. D. (2007). The role of working memory representations in the control of attention. Cerebral Cortex, 17, i118-i124.

Woodman, G. F., Vogel, E. K., \& Luck, S. J. (2001). Visual search remains efficient when visual working memory is full. Psychological Science, 12, 219-224.

\section{NOTE}

1. Jolicœur and Dell'Acqua (1998, 1999; see also Dell'Acqua \& Jolicœur, 2000; Jolicœur, 1999) reported significant interference between speeded auditory tasks and unspeeded visual judgments. The locus of interference in these cases, however, appears to lie in the process of consolidating visual information into short-term memory, beyond the stage of visual perceptual processing.

(Manuscript received August 11, 2009; revision accepted for publication October 11, 2009.) 